\title{
Tevatron faces final curtain
}

\author{
Particle accelerator to be switched off this year, as lack of funds spells the end for \\ US bid to capture Higgs particle.
}

\section{BY EUGENIE SAMUEL REICH}

$\mathrm{D}$ epending on who you talk to, it is either a disappointing blow or a clean break heralding an exciting new era. After much debate, officials at the US Department of Energy's Office of Science revealed this week that they have decided not to extend funding for the Tevatron, the proton-antiproton collider at Fermilab in Batavia, Illinois, by an additional three years. The decision means that the first glimpse of the long-predicted Higgs particle, thought to endow other particles with mass, will probably be achieved by the Large Hadron Collider (LHC) at CERN, Europe's particlephysics lab near Geneva in Switzerland.

The decision was explained in a letter sent on 10 January by Bill Brinkman, director of the Office of Science, to Melvyn Shochet, a physicist at the University of Chicago, Illinois, and chairman of the energy department's High Energy Particle Advisory Panel (HEPAP). In October 2010, with the LHC suffering from delays, HEPAP had recommended that the US machine be extended beyond its planned 2011 closure if extra funding of US $\$ 35$ million could be found. It couldn't, says Brinkman. "Unfortunately, the current budgetary climate is very challenging and additional funding has not been identified," he writes in his letter. $\mathrm{He}$ adds that the Tevatron will shut down this year as planned.

The decision is a blow for the 1,200 physicists who work on experiments at the Tevatron, the world's second most powerful accelerator after

"I'm glad they
made a clean,
unambiguous
decision. One
concern was
a drawn-out
process."
the LHC, as well as for particle-physics theorists at Fermilab and elsewhere who would have enjoyed working with the data. "I feel disappointed," says Pier Oddone, Fermilab's director. "We all would have wanted to see the Tevatron continue."

Rick Van Kooten of Indiana University Bloomington, who recommended the extension to the Tevatron in his role as chairman of Fermilab's Physics Advisory Committee, says he's also disappointed at the decision, but is convinced that sincere efforts were made to find the funding.

Leaders of Tevatron experiments made the case for extension last year, arguing that the machine's smooth running, combined with

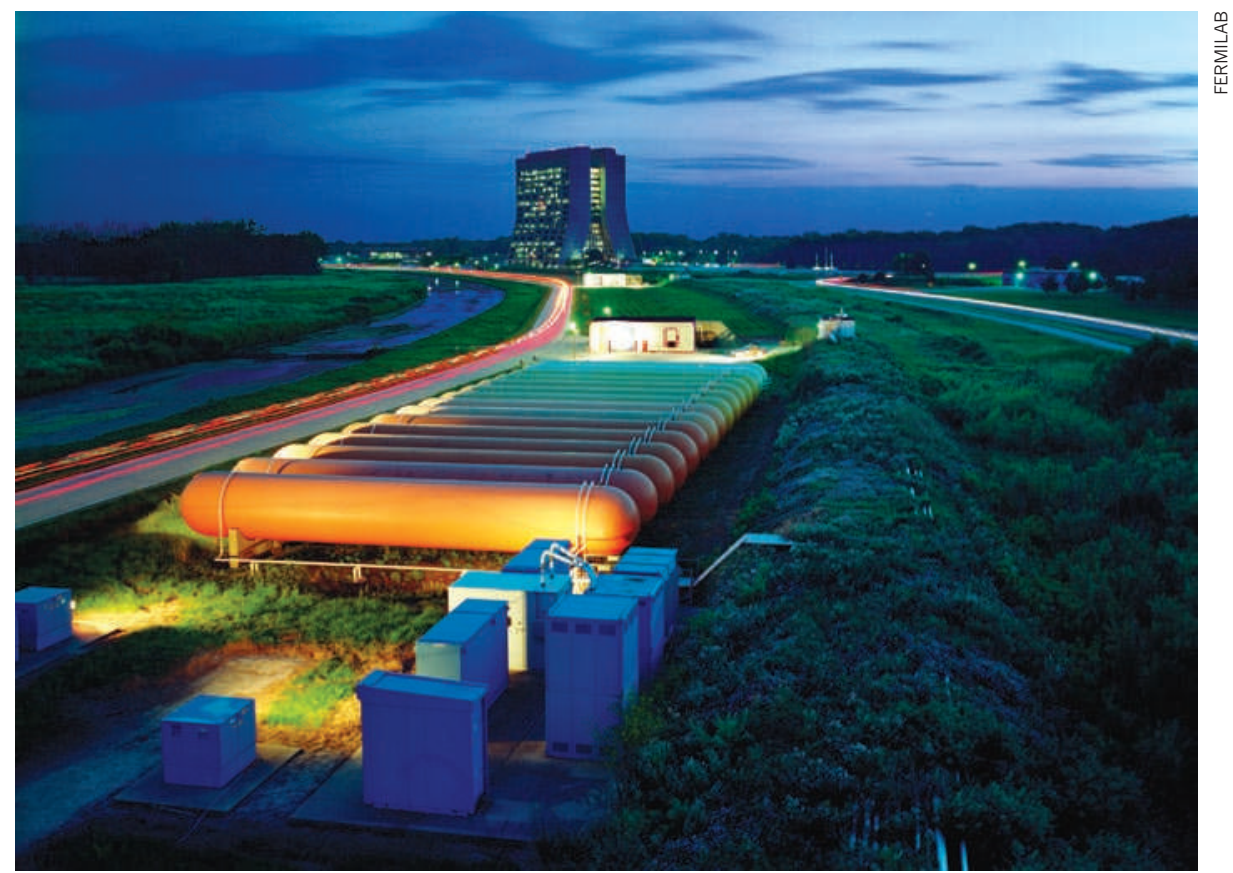

The Tevatron's 6.3-kilometre-long ring will stop colliding particles this year rather than in 2014.

technical problems at the LHC, provided the Tevatron with a shot at discovering the Higgs, and the glory that would go with it. The two advisory panels also agreed that the physics case was compelling. But Charles Baltay, a physicist at Yale University in New Haven, Connecticut, and the chairman of P5, the HEPAP subpanel that considered the request, says this week's decision not to go ahead is consistent with the $\mathrm{P} 5$ recommendation that the Tevatron extension be funded only by adding to the high-energy physics budget, and not by taking funds away from other US particlephysics experiments. "I'm glad they made a clean, unambiguous decision," he says. "One concern was a drawn-out process."

In a way, the decision can be seen as a vote of support for a programme mapped out by P5 in 2007 and 2008. According to that programme, the United States should concede the realm known as the 'energy frontier', involving the highest-energy particle collisions, to the LHC, instead focusing its domestic efforts on 'the intensity frontier', achieving the greatest number of particle collisions per second. The latter is ideal for studying rare processes, and so $\mathrm{P} 5$ recommended investment in a slew of experiments to do just that. They include Fermilab's Mu2e, which will study the rare decay of muons to electrons, and NOvA and the Long Baseline Neutrino Experiment, which will pin down the mass and other properties of elusive neutrinos, expected to have relevance for explaining the asymmetry between matter and antimatter in the Universe.

Mark Messier of Indiana University Bloomington, co-spokesman for $\mathrm{NOvA}$, says the decision makes things easier for experiments that would have competed for resources had the extension been approved. "We're back on the original course. There's something that we now don't have to deal with," he says. The Tevatron extension would have delayed the start of NOvA, because the neutrino experiment is designed to use the Tevatron's cast-off recycler, a particle-storage ring.

Robert Roser, co-spokesman for one Tevatron experiment, the Collider Detector at Fermilab, says that physicists on the experiment will now focus on ensuring the most successful end to the current run. "We raised the bar for the LHC. We're very proud of our accomplishments." The analysis of data from the machine is expected to continue for the next two to three years.

Oddone says that the exact switch-off date will become clear once the US Congress passes the fiscal year 2011 budget. 


\section{Missing part delays space mission}

Schedule slips for European-led effort to blaze a trail for gravitational-wave detection.

\section{BY EUGENIE SAMUEL REICH}

$\mathrm{F}$ or Stefano Vitale, a principal investigator on the LISA Pathfinder mission, the situation is excruciating. Nearly all the instruments for the $€ 300$-million (US\$400million) spacecraft have been delivered for what was originally to have been a launch this year. But delays have pushed that target to 2013 and possibly later, with everything now held up by a small but crucial component. "All the rest is waiting for one part. It's heartbreaking," says Vitale, a physicist at the University of Trento in Italy.

It is a rougher-than-anticipated start for a mission that was created to find obstacles. LISA Pathfinder is a European-led test of the technology needed to run the Laser Interferometer Space Antenna (LISA), an ambitious effort to detect gravitational waves from sources in the distant Universe. Scientists hope that LISA can achieve this by measuring the precise separations between three pairs of masses free-floating inside three spacecraft positioned 5 million kilometres apart. The technical challenge along with the estimated cost of LISA ( $€ 1$ billion to $€ 2$ billion) made a precursor mission a necessity. If LISA Pathfinder encounters significant problems it could sow doubts about the overall effort.

LISA Pathfinder is not expected to detect gravitational waves, but it must deploy and measure the relative positions of two test masses with sufficient precision for LISA to move forward. The missing piece of the mission is part of a 'caging mechanism' consisting of two sets of eight fingers that will hold the two 1.96-kilogram gold-platinum masses during launch, and then, once the spacecraft reaches its orbit at the L1 Lagrangian point where the gravitational pull of Earth and Sun are balanced, delicately release them. The masses will then float freely inside their separate compartments while the spacecraft uses electrical microthrusters to maintain its position so precisely that the masses do not hit the sides of their containers.

The exacting requirement for a mechanism that can hold the masses firmly enough to withstand a force of 2,000 newtons but still release them without imparting a velocity of more than 5 micrometres per second (18 millimetres an hour) lies at the heart of the delay. A first prototype of the motor powering the fingers failed key tests, prompting the European Space Agency (ESA) to set up a task force to look into the problem. The motor is now being redesigned from scratch. "Little by little, the launch date is slipping," says Pierre Binétruy

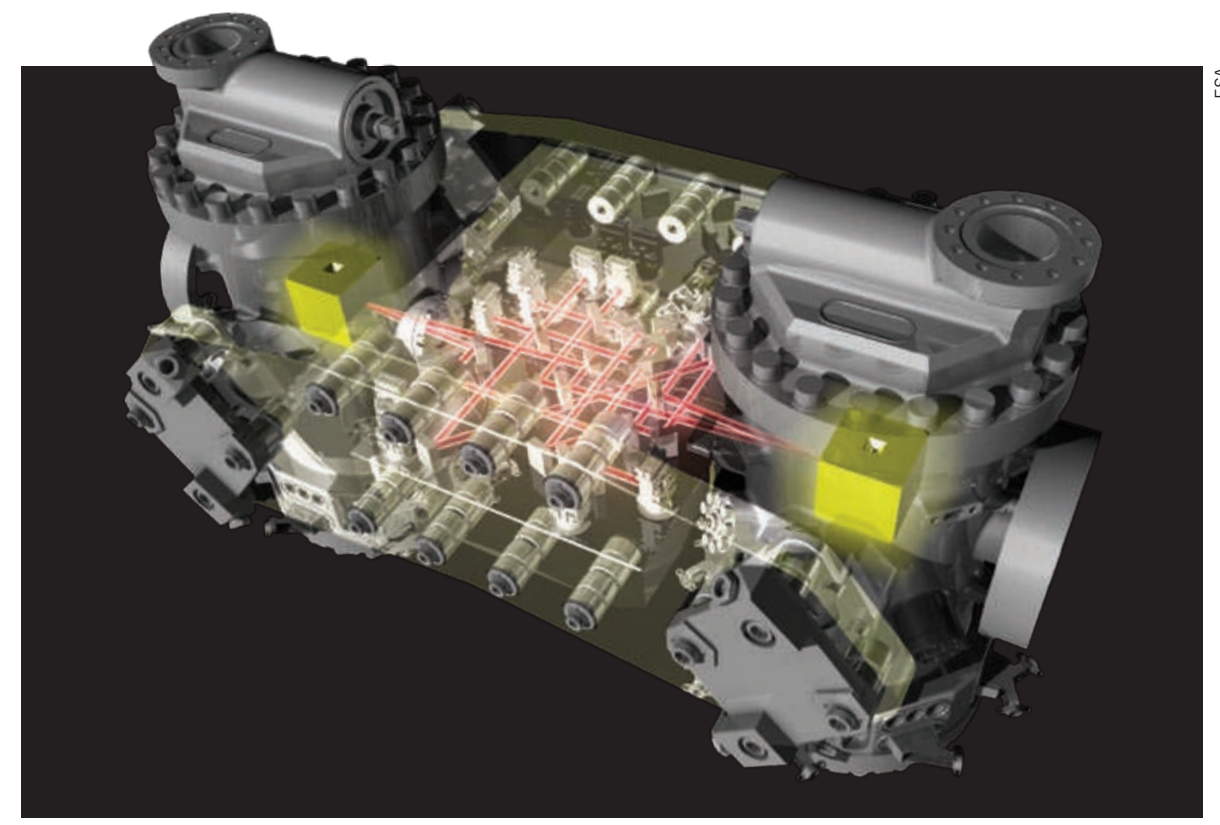

The LISA Pathfinder is missing the mechanism to hold two masses (yellow cubes) in place during launch.

of Paris Diderot University, a physicist on the LISA international science team.

Scientists on the mission say that the important thing is to learn from the delay, to avoid similar problems on LISA. With LISA Pathfinder, ESA initially followed a conventional model for managing space missions, assigning science research groups outside the space agency to design the payload - including the caging mechanism - while industrial partners designed the spacecraft itself. But designers found that the spacecraft was operationally indistinguishable from its science payload, because the positioning of the masses inside it is coupled closely to the craft's ability to keep its place in space using the microthrusters. ESA then took on the design of the caging mechanism together with a contractor, Thales Alenia Space in Milan, Italy, which was unable to comment before Nature went to print.

On 10 February, the ESA Science Programme Committee is expected to assess options for the new design and chart a path forwards.

Last August, the Astro2010 decadal survey of the US National Academy of Sciences ranked participation in LISA among its top priorities, above a competing project, the International $\mathrm{X}$-ray Observatory (IXO). But that recommendation assumed a successful LISA Pathfinder. Xavier Barcons, a physicist at the Cantabria Institute of Physics in Santander, Spain, who works with IXO, says the problems on Pathfinder call into question the decision to rank it higher than his project. "We also have technical difficulties but we've mastered the basics. LISA is a completely new adventure," he says.

He says that it is not clear whether LISA can fly by 2025, as the decadal survey assumed. But Fabio Favata, head of ESA's science coordination office, says that by uncovering problems early, LISA Pathfinder could help LISA avoid delays. "The present situation, although unfortunate, does emphasize the importance of pathfinding," he says. -

\section{CORRECTIONS}

The timeline in the News story 'Cancer trial errors revealed' (Nature 469, 139-140; 2011) stated that Harold Varmus asked the Institute of Medicine to review Duke University's trials in June 2010. He made this request in July 2010.

The News story 'Tevatron faces final curtain' (Nature 469, 141; 2011) states that the Mu2e experiment will study the decay of muons to electrons. In fact, it will look for evidence of neutrino-less conversion of muons to electrons.

The story 'Science fortunes of Balkan neighbours diverge' (Nature 469, 142-143; 2011) wrongly referred to the Romanian Academy of Sciences - it should have said the Romanian Academy. Also Bulgaria cut the budget of its Academy of Sciences by $38 \%$ to 60 million leva (US $\$ 40$ million), not 75 million leva as stated. 